This electronic prepublication version may contain typographical errors and may be missing artwork such as charts, photographs, etc. Pagination in later versions may differ from this copy; citation references to this material may be incorrect when this prepublication edition is replaced at a later date with the finalized version.

\title{
Locating Library Items by GIS Technology
}

\author{
Jingfeng Xia
}

\begin{abstract}
This article identifies a common problem in many academic libraries: users having difficulties finding library items. It suggests that a mapping system may help solve the problem if the difficulties stem from users who do not understand floor structures of the library or classification of the collections. The technology of Geographic Information Systems (GIS) is proposed to build the system because of its ability to analyze spatial data of collection locations and dynamically present the analytical results in maps of varied detail. Such a GIS item-locating system could be integrated into current online catalog systems so that users would have search results in both textual display and visual demonstration. The article advocates that libraries take advantage of GIS for its inexpensive development and simple implementation. [Article copies available for a fee from The Haworth Document Delivery Service: 1-800-HAWORTH. E-mail address: <docdelivery@haworthpress.com>Website: <http://www.HaworthPress.com> ( 2005 by The Haworth Press, Inc. All rights reserved.]
\end{abstract}

KEYWORDS. Item location, maps, GIS, online integration

\section{PROBLEMS IDENTIFIED}

One of the common problems in the utilization of library collections is users' inability to find materials. This is particularly prevalent in the academic library environment where collections are large and stored in

Jingfeng Xia is in the School of Information Resources and Library Science, University of Arizona. He can also be reached by e-mail: jxia@canada.com.

Collection Management, Vol. 30(1) 2005

Available online at http://www.haworthpress.com/web/COL

(C) 2005 by The Haworth Press, Inc. All rights reserved.

doi:10.1300/J105v30n01_07 
varied physical locations. It may be understandable if the users cannot find a specific item because it is misplaced or being used by someone else. However, in many situations they have difficulties finding an item even though the item is in the correct place.

Reasons for this confusion are many, but mostly it is caused by the way libraries manage their collections or by the users themselves. Libraries may have not organized their shelves logically or may not offer adequate signage or labeling. Such problems can be solved once they are identified. On the other hand, difficulties finding items due to users' lack of knowledge of collection organization are more intractable.

Currently, a large majority of academic libraries use the Library of Congress (LC) classification system to organize their collections. This system is fairly straightforward to those with experience in exploring library resources. For many university freshmen and inexperienced users, however, it may not be as easy to understand. For example, the Cutter Rules in the LC system confuse some people and lead them to pass over the correct stack location, because they may not recognize that these numbers are decimal values. Further complications may result if double Cutter numbers are assigned.

It is noteworthy that this problem is by no means infrequent in academic libraries. It often emerges among the top concerns for both librarians and users. For example, a survey conducted among undergraduate students in the Central Missouri University Library revealed that the number one function for reference librarians, from the perspective of the student, is to direct library patrons to physical resources (Jenkins, 2001). In many academic libraries, this is one of the "high problem areas" and demands ongoing attention.

Usually, users who are not able to find an item (e.g., a book) will ask for help from reference librarians. The latter, however, are often too busy to offer such help if they are asked to physically retrieve individual items. Some users may even be hesitant to ask for help owing to a variety of psychological reasons (Currie, 2002). As a result, library collections may be unable to reach the goal of efficient utilization, and frustrated users may accumulate dissatisfaction with library services.

Many libraries have already identified this issue as a serious problem and developed some solutions. The most common solution is to organize instructional sessions to teach new students how to use library resources (Hull and Lawton, 2001; Whitmire, 2001). Occasional workshops are especially popular, although semester-long for-credit library methods courses are also available at some places (e.g., Blakeslee, 1998). Unfortunately, such instruction can only cover a small percent- 
age of the student population in practice. The problem of users unable to find items in library collections still deserves further consideration for workable solutions.

\section{SOLUTIONS PROPOSED}

Academic libraries have as their goal to "create students who can use the library's resources independently" (Quinn, 1997, 359). To reach this goal, a good solution is one that would help users visualize information about the collection so they can quickly grasp where individual items are located. A feasible format for such visualizations is "the map".

Nevertheless, controversies exist about where to set maps and what kind of maps are appropriate, as well as how to implement the placement and updating of maps. In answering the question of what kind, it is actually easy to figure out if we remain cognizant of how confusions are generated for inexperienced students, unaware of collection sectors and unfamiliar with call numbers. For example, if books with call numbers GN are stored on the third floor of a library building in Section D (supposing that the third floor has five space segments, each of which has its own stacks, study areas, and other facilities), a student who looks for a book by call number GN100 .T12345 1900 may first be puzzled by the floor structure: where are Sections A, B, C, D, and E? As soon as he or she approaches Section D of the third floor, the second puzzle that may come up is which shelf holds this book, because one shelf-range ends with call number GN100 .T2 and the next shelf-range starts from call number GN100 .T3. If the student is fortunate enough to locate the correct section of shelves, then it may be unclear precisely where the book belongs, because he or she does not really understand the LC system.

This scenario suggests at least three maps for each item: floor structure, shelf-ranges, and bookshelf layers. This recalls the previous question about where to place these maps. The first map can simply be erected at the entrance of each floor, while the latter two are associated specifically with individual records and, thus, require dynamic presentation. The best place to present these two maps (as well as the map of the floor structure) is on the online catalog screen where users see bibliographic records for searched items. Ideally, the catalog system is able to provide readers with not only bibliographic records of an item, but also maps to show the exact location of this specific item in the library. The maps will guide the users to the correct destination. 
Figures 1-3 illustrate how textual records might interact with three location maps in the online catalog interface. Figure 1 is a screen capture for the search result of a book in an imaginary university library. The user finds bibliographic records of the book at the upper portion of the screen and a floor map of the book at the lower screen. This map is clear enough to give one a quick view of the floor location of the book as well as directions to the area where collections are located.

The floor map is expandable. Upon clicking on it, the screen presents a new window with another map that displays shelf-ranges in the collection sector among which the book is shelved. Figure 2 uses a different color to guide the user to the particular shelf-range and section where the book resides. Note that each shelf-range is proposed to have a sequential number as label for clear identification. The eye-catching label is better attached at both ends of a shelf-range (conventional call number identifiers can remain in the same place), enabling users to navigate shelf-ranges without difficulty.

Figure 3 can be displayed side by side with Figure 2 on the same screen. Alternatively, it can be brought up by clicking Figure 2. Its purpose is to tell the user on which level of the bookshelf this book is placed. It should be noted here that the position of the colored book on the layer does not represent its precise position. The figure is only for visual demonstration. Still, this type of detailed visual presentation offers users an excellent chance to find what they are looking for and avoid confusion.

\section{GIS INVOLVEMENT}

Making maps and catalogs an integrated online system requires the implementation of a dynamic processing mechanism. Maps would have to quickly respond to users' searches. Current OPAC systems do not have the capability to handle the integration. Computer programming may make it work, but would be very complex and costly. An excellent solution is the technology of Geographic Information Systems (GIS).

Designed specifically for geographical applications, GIS is better than any other software for manipulating and presenting maps. It has the power of connecting maps to databases and analyzing spatial data dynamically. Upon completion of spatial analysis on data (or updates of data), the results can immediately be reflected on GIS-produced maps. The scalability of GIS technology also ensures an internet interaction. Such abilities provide it with advantages in the development of a GIS 
FIGURE 1. The result of an onlince catalog search in an imaginary university library. The physical location of the searched item is shown on a floor map.

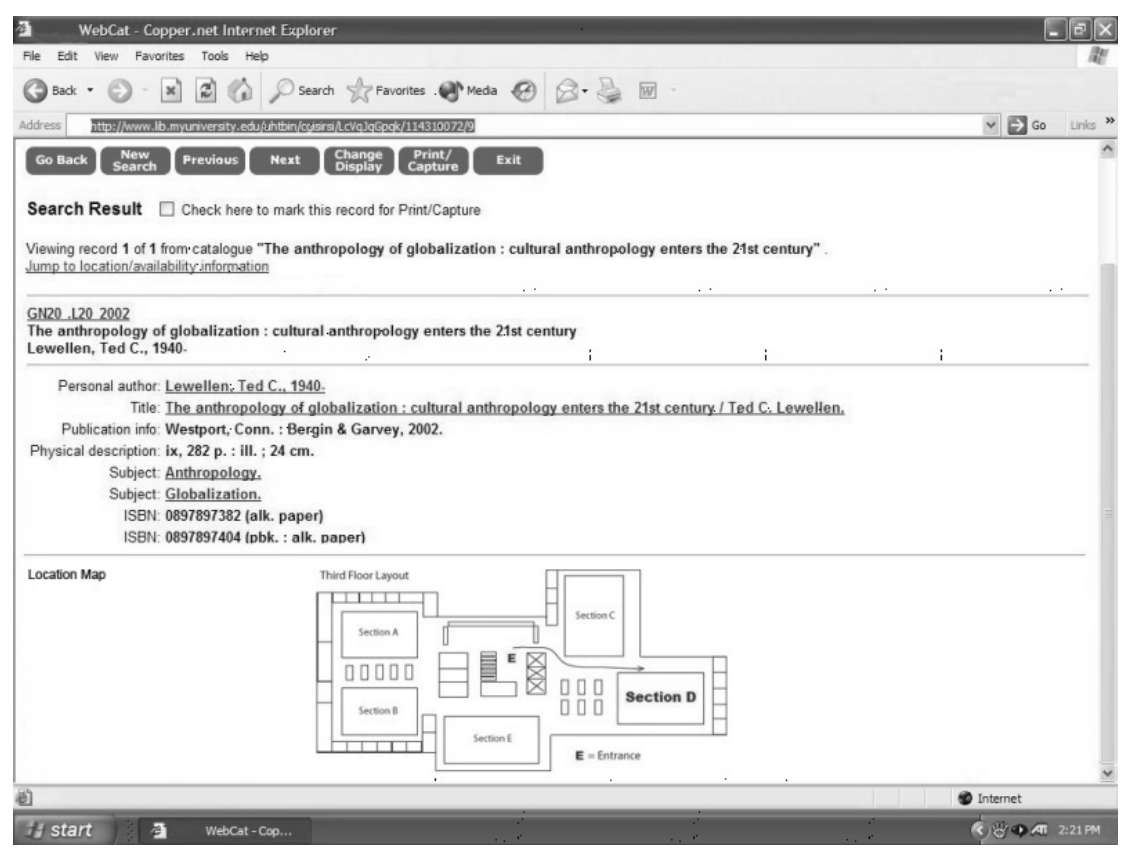

item-locating system (GISils) for libraries. Most academic libraries have already incorporated some GIS products among their services, making the introduction of GIS technology into this development inexpensive. Moreover, it is relatively easy to develop the system, and any GIS software, such as ArcView or ArcGIS, can be an excellent candidate.

There are three steps in developing GISils: (1) creating maps for floor layouts, shelf-ranges, and bookshelves; (2) designing a database that contains bookshelf information and connects to existing cataloging databases; (3) collaborating with the structure of online cataloging. Below are highlights of a development plan that may be modified by any library with an interest in pursuing this concept.

\section{Maps}

Floor maps created in GIS are for demonstration only so that structure details may not be required. One map for a floor is generally suffi- 
FIGURE 2. The 3-D view of some shelf-ranges in a collection sector. The bookshelf on which the searched item is stocked is highlighted with a different color.

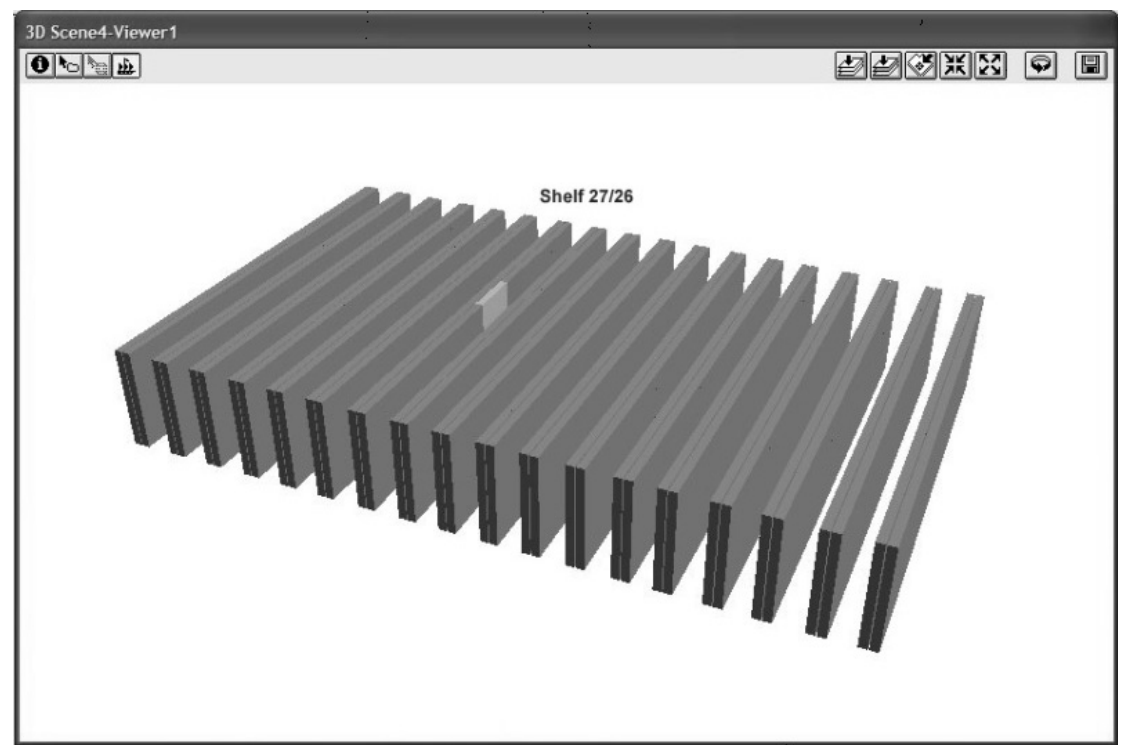

cient although some variations exist. Most current library catalog systems are able to automatically display the location of an item in wording, indicating that their databases already contain certain location information. One of the goals for developing such a GIS system is to use visual presentation to replace the text.

The maps can be drawn based upon the building blueprints of a library by using a computer-aided drawing device-AutoCAD. Alternatively, regular digitizers connecting to a GIS product can also be utilized. Each feature on the GIS maps, including polygons, points, and lines, will be established with its own identification and related feature data. Polygon, which can represent shelf-ranges, bookshelves, and layers, is the most important feature in this case. Geo-referencing, however, is not necessary because spatial precision is not as critical as on conventional maps.

Shelf-range maps represent the physical conditions of each collection sector, which is usually one space segment of a floor. These maps are subdivisions of the floor layouts with a zoomed-in appearance. The 
FIGURE 3. The front view of a shelf-range. The searched item is colored on a particular layer where it belongs to.

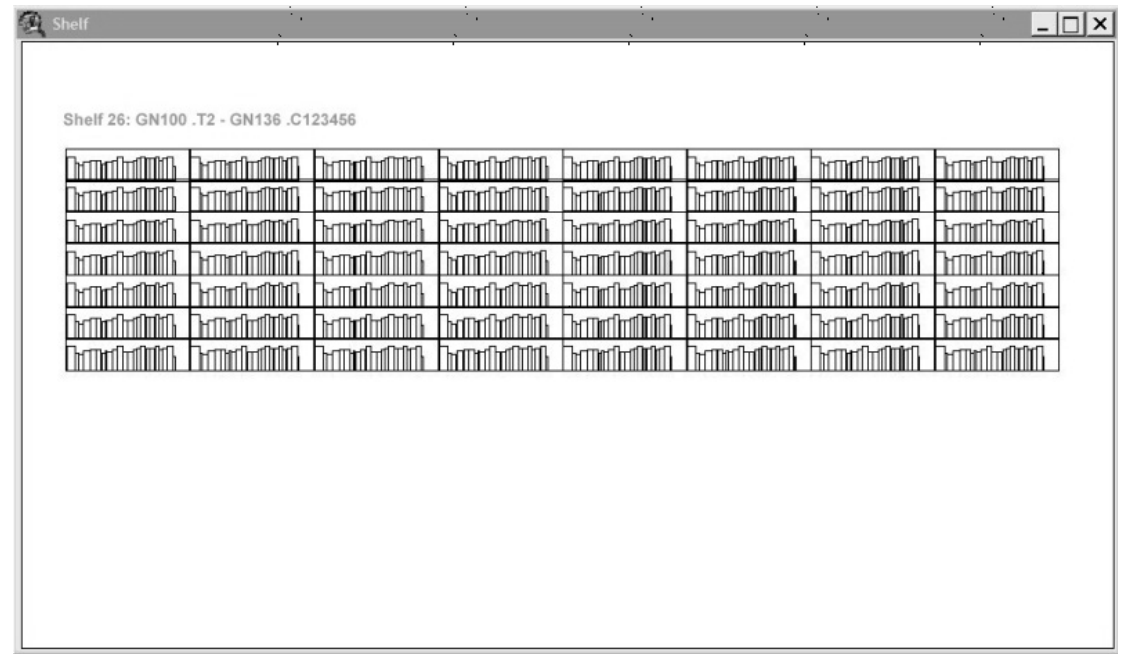

number of such maps required for the system depends upon the structure of a library building. Usually, less than a dozen are adequate. Both 2- and 3-dimensional presentations work well, although 3-D maps offer better comprehensibility. It is a simple task for GIS software to generate a 3-D scene from a 2-D map.

Only one bookshelf map is required to stand for all bookshelves unless the library has different types of structures for its bookshelves. Books shown on these shelves are for demonstration (e.g., Figure 3 ) and do not represent actual size and ranking. When creating this map with GIS, each book corresponds to a polygon, which may not need associated feature data.

\section{Databases}

In order to integrate the GIS technology into existing online catalog systems, a new database would need to be designed and connected to the GIS product. It will contain several database tables that hold geospatial data for every feature in the above-mentioned maps, for example, their coordinates. The tables also store other data necessary for the dynamic 
analysis and display of individual library items. For this specific project, the following information is fundamental:

- Floor number (e.g., First Floor, Second Floor . . .)

- Section identification (e.g., 3A, 3B, 3C ...)

- Shelf-range label (e.g., Shelf 25, Shelf 26, Shelf 27 . . .)

- Bookshelf layer rank (e.g., Layer 1 for the top layer, Layer 7 for the bottom layer)

- Start/End call numbers for each bookshelf layer

- Start/End call numbers for each bookshelf

- Start/End call numbers for each shelf-range

- Start/End call numbers for each collection sector

- Individual call number

The last one serves as a relational key in each database table linking to its counterpart in the catalog databases upon which both bibliographic records from the catalog databases and location information from the GIS database for any particular item can be returned simultaneously to respond to an online search. Such data can be updated by importing from existing catalog databases, which can be an easy process.

The controversial part of working with the database is how to deal with the Start/End call numbers for each shelf. The logic requires use of the call numbers of the first and last book on a shelf to identify this shelf. Whenever a book is searched, its call number will be assessed against shelf identifiers in SQL queries to determine on which shelf the book exists. Then, GIS will highlight it at the corresponding location on a map as shown in Figure 3.

Updating such information could be the most tedious work in the development of this system. For example, suppose a library has four floors and each floor is divided into three collection sectors. Further suppose that there are one hundred shelf-ranges standing in each sector and each shelf-range is composed of ten book sections. If one section has seven shelves, the total number of shelves could be as many as 7,000 $(7 \times 10$ $\times 100)$ for a sector, $21,000(7 \times 10 \times 100 \times 3)$ for a floor, and 84,000 $(7 \times 10 \times 100 \times 3 \times 4)$ for the library. A total of 84,000 records would require Start/End call numbers. Furthermore, whenever library assistants shift books from shelf to shelf, the change will have to be updated. If this task is considered to be too onerous, the presentation of book location at the shelf level could be sacrificed. 
As soon as the database is completed, it would be integrated with the maps into GISils, and saved on the same computer server that holds the catalog databases.

\section{Online Interaction}

The display of bibliographic records in the OPAC for an item will remain the same. Map presentation needs extra work, however. Certain programming codes must be installed on the computer server in order to call GISils for execution. According to the programming experience of the author, the codes can be very simple to develop. The whole process of performing GISils online search is outlined as the follows.

1. After the user clicks the Search button or strikes the Enter key for a search, the online catalog databases begin to query associated data and display textual results onto the screen. This is the process used to search online catalogs today.

2. At the same time, programming codes are triggered that identify the call number and pass it as input parameter to GISils.

3. GISils looks for this item in its own database and, upon an analysis of the data, highlights its location with color on corresponding maps.

4. The maps are sent back to the web server that displays the floor map underneath the bibliographic records on the same screen.

5. Other maps wait on the web server for user's further clicking.

Thanks to today's powerful computer and advanced GIS technologies, the whole process can be accomplished in a couple of seconds. There is no significant delay over a current online search. Users should not notice the integration of GISils behind the scenes.

\section{CONCLUSION}

GIS technology has been proposed as a solution for helping users to locate library items. Through integration into current online catalog systems, GIS can become an automated system that is able to quickly find the location of a searched item, highlight the location on different maps, and present the maps to users on the catalog screen. The system will

guide library users to the correct locations, thus improving the effectiveness of collection utilization. 
Nonetheless, GIS has its limitations in such development. To be specific, it cannot tell users where to find an item if the item is not in its correct location or is being used by somebody else (and not checked out). It may be possible to combine it with some other technologies that have the capability of tracing items, such as Radio Frequency Identification. One of the advantages that GIS technology can provide is low cost and easy development. At this stage, the use of GIS as proposed here, is merely a concept. It is possible that some academic libraries can implement it in their management systems in the near future.

\section{REFERENCES}

Blakeslee, S. (1998), "Librarian in a strange land: Teaching a freshman orientation course," Reference Services Review, Vol. 26, pp. 73-8.

Currie, C.L. (2002), "Difficult library patrons in academe: It's all in the eye of the beholder," The Reference Librarian, Vol. 75/76, pp. 45-54.

Hull, T.L. and K.A. Lawton (2001), "The development of a first-year student library instruction program at Duke University," The Reference Librarian, Vol. 73, pp. 323-36.

Jenkins, S. (2001), "Undergraduate perceptions of the reference collection and the reference librarian in an academic library," The Reference Librarian, Vol. 73, pp. 229-41.

Whitmire, E. (2001), “A longitudinal study of undergraduates' academic library experiences," Journal of Academic Librarianship, Vol. 27, No. 5, pp. 379-86.

Received:

Reviewed:

Revised and Accepted: 